\title{
A Kinect Rehabilitation Training System Based on Improved DTW-RANSAC Evaluation Model
}

\author{
Xiaonan Shi ${ }^{1 *}$, Zitong Wang ${ }^{2}$ \\ ${ }_{1}^{1}$ Xi'an University of Science and Technology, Shaanxi, China. \\ 2 Xi'an, Shaanxi Province, China Xi'an University of Science and Technology, China. \\ * Corresponding author. Tel.:+8613149183189; email: 514861529@qq.com \\ Manuscript submitted July 16, 2017; accepted September 8, 2017. \\ doi: 10.17706/jsw.12.12.934-944
}

\begin{abstract}
In view of the application of Kinect equipment in medical rehabilitation, the training plan of Kinect rehabilitation training system based on DTW-RANSAC evaluation model is put forward. The patient's bones movement information is collected by the Kinect device and the result is sent to the server through the client. The data of the bone motion data is stored on the server side and compared with the standard template information according to the DTW-RANSAC evaluation model algorithm and the action evaluation results are sent to the physician client to assist the patient in giving the patient a further treatment plan. Experimental results show that the system can accept and recognize the action, to achieve customer rehabilitation training, communication between doctors and patients and other functions, reduce the pressure of rehabilitation training doctors, there is a certain innovative good practical.
\end{abstract}

Key words: Kinect, rehabilitation training, DTW-RANSAC algorithm, skeletal motion information.

\section{Introduction}

Kinect is a research and development and commercialization of human-based interactive human-computer interaction device, which uses real-time dynamic skeletal tracking, image recognition, microphone input, voice recognition and other functions to allow users from the traditional game controller shackles, through the body to control the game The With the release of Kinect for Windows SDK, more and more areas are using Kinect to develop related applications such as interactive education, sports training, dance games and so on. At present, there are many foreign scholars to start the application of Kinect [1-5]. Domestic scholars such as Qin Chao Long ${ }^{[6]}$ and so on using Unity3D combined with Kinect manufacturing rehabilitation training robot to help patients for rehabilitation training, Yang Wenlu ${ }^{[7]}$ and so on using fuzzy C-means clustering algorithm to extract the key posture action, and then use Euclidean distance to match, To complete the rehabilitation training, Yang Yi [8] the use of virtual reality and Kinect equipment to build a dynamic real-time rehabilitation training software system, Luo Yuan [9] based on Kinect sensor design and implementation of intelligent wheelchair gesture control system, Zhou Jin [10] Rapid reconstruction of three-dimensional human body model for subsequent rehabilitation training design.

At home and abroad on the Kinect somatosensory rehabilitation research focused on hemiplegia, stroke, traffic accidents caused by the active function of patients with active rehabilitation training and treatment. However, the existing Kinect somatosensory rehabilitation system problems first of all, the patient's human body target capture is not accurate, mistakenly identify the problem more; followed by the patient action and standard action matching efficiency is low, matching inaccurate lead to slow progress in rehabilitation 
training. For the second question, many rehabilitation systems use DTW (dynamic time regularization) algorithm to match the action.

But the classic DTW has several limitations [11]:

(1) Efficiency: The efficiency of the DTW algorithm is related to the test data establish and the ratio of the inner point of the class. So the success rate of some actions is lower.

(2) Accuracy: The DTW algorithm calculates the data from the economic angle to select the smallest subset, but obtains generally is the best result. Before uses, these results must undergo the refinement processing.

(3) Degradation: The DTW algorithm uses false attempts to maximize the objective function: "If there is a class of external point in the selected minimum test set, in this case, the calculated parameter obtains the target function (the number of test data midpoint) is often less." "But this is seldom the case when degradation occurs.

DTW Ransac is an algorithm that can get effective sample data, and when test data and standard data are sent to DTW and RANSAC, two similarity evaluation results are produced. In this paper, the doctor's judgment can be added to the system through the DTW RANSAC algorithm, which can enhance the reliability of the system and provide doctors with more correct medical data.

\section{The Design of Kinect Rehabilitation Training System Based on DTW RANSAC Algorithm}

This article designed Kinect-based rehabilitation training system is the basis of algorithm operation, the system operation of the important steps are as follows:

Step 1: Obtain skeletal motion frames from Kinect;

Step 2: Motion recognition by mean Hausdorff algorithm and HMM algorithm;

Step 3: Use the improved DTW-RANSAC dual algorithm to compare the patient action with the standard action;

Step 4: Reveal the results of rehabilitation training.

\subsection{Skeleton Information Data}

The skeletal data obtained by Kinect acquisition are generated by Skeleton Stream, where each frame of data to SkeletonFrame is a collection of skeletal objects. The SkeletonFrame contains an array of structures for skeletal data, where each skeletal information is tracked by the bone recognition system. Both skeletal coordinate data and skeletal joint data are saved in an array of skeletal information. Kinect can identify 25 joints, each joint has a unique identifier corresponding to three-dimensional coordinates, such as the Hand Right, the Knee Left, Spine and so on. The corresponding processing is then started: the skeletal frame data are stored in the NUI_SKELETON_FRAME structure.

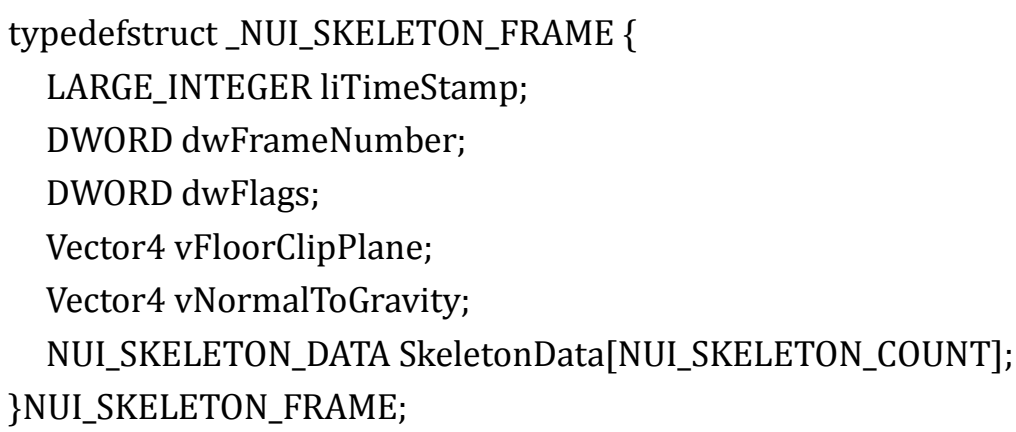

\subsection{Action Recognition and Matching Algorithm}

Due to the immediacy of the action. It is necessary to compare the action at all times. That is, after each 
frame of skeletal information data has been collected and standardized, it is compared with the standard action and the evaluation given to the post-system is fed back to the user. In this paper, the action recognition algorithm based on the mean Hausdorff distance and the recognition algorithm of the human body action based on the hidden Markov method are used to match the standard action data and the patient activity data with the improved DTW-RANSAC evaluation model. Based on the reliability evaluation Skeleton reconstruction method, to solve the shortcomings of Kinect skeleton data accuracy.

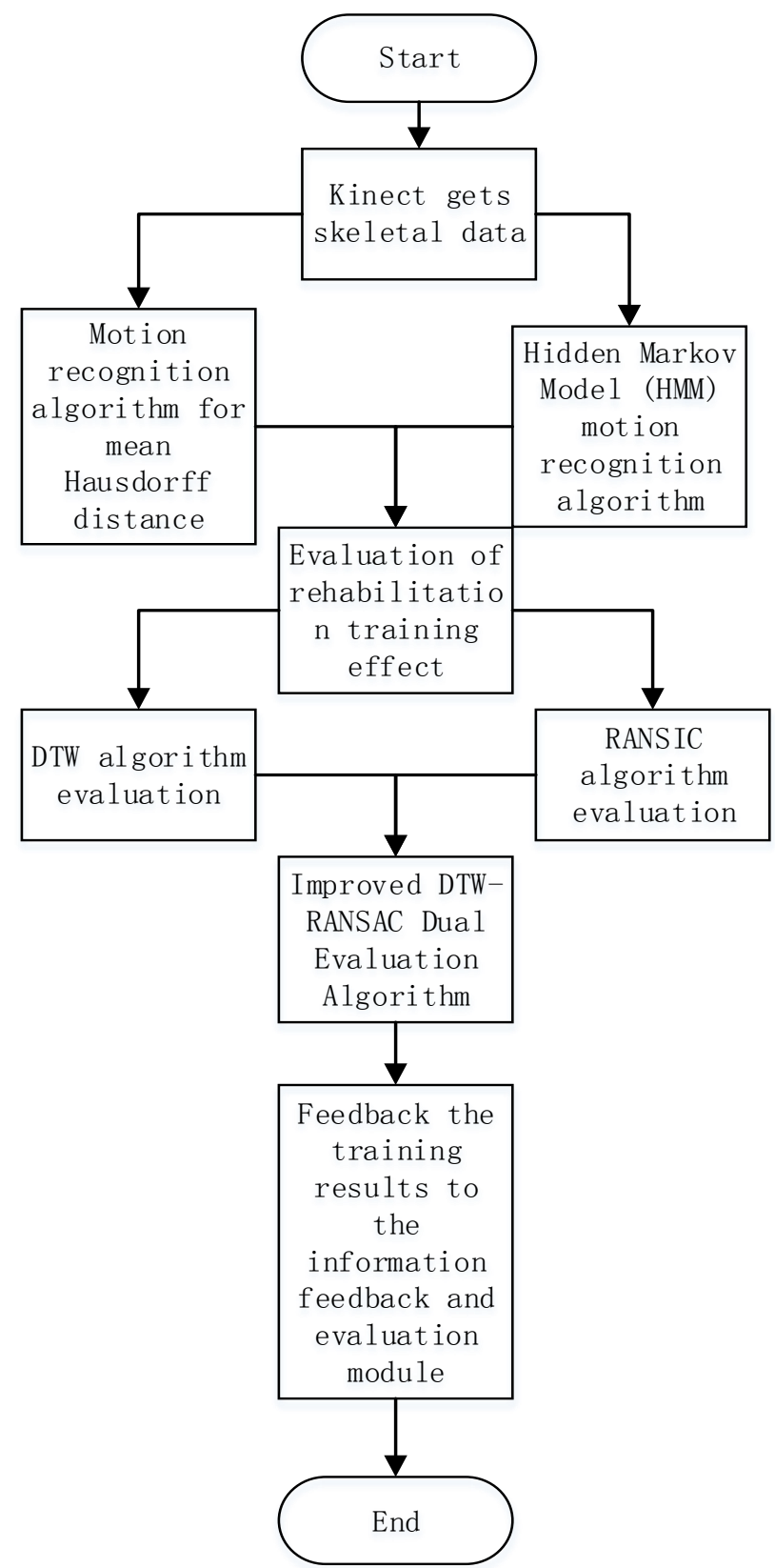

Fig. 1. Kinect - based training system for rehabilitation training system.

\subsubsection{Motion recognition algorithm based on mean Hausdorff distance}

The Hausdorff distance is defined as a set of two points $A=\{a 1, a 2, \ldots, a m\}, B=\{b 1, b 2, \ldots, b n\}$, a measure of the similarity between A and B, So we can use the formula to calculate the Hausdorff distance between two points: 


$$
\begin{gathered}
\mathrm{H}(A, B)=\max (h(A, \mathrm{~B}), h(B, A)) \\
\mathrm{h}(\mathrm{A}, \mathrm{B})=\max _{a \in A} \min _{b \in B}\|a-b\|, h(B, A)=\max _{b \in B} \min _{a \in A}\|b-a\|
\end{gathered}
$$

The disadvantage of Hausdorff algorithm is easy to be disturbed by noise, produce the wrong result, affect the effect of recognition, in order to solve this problem, the part Hausdorff distance which Dubuisson et [13] put forward, effectively reduces the error, the partial Hausdorff distance is expressed as:

$$
\begin{gathered}
H_{L K}(A, B)=\max \left\{h_{L}(A, B), h_{K}(B, A)\right\} \\
h_{L}(A, B)={\underset{a \in A}{L}{ }^{t h}}_{b}(a), h_{k}(B, A)={\underset{b \in B}{L}{ }^{t h} d_{a}(b)}^{t}
\end{gathered}
$$

Hausdorff distance may be the first error match the situation is the matching target data is blocked by other things or contain noise, so in order to improve the matching accuracy, Dubuisson et al [13] people will define the mean Hausdorff distance (MeanHausdorffDistance, MHD), expressed as:

$$
\begin{gathered}
\mathrm{H}(A, B)=\max (h(A, \mathrm{~B}), h(B, A)) \\
\mathrm{h}(A, B)=\frac{1}{r} \sum_{a \in A} \min _{b \in B}\|a-b\|, h(B, A)=\frac{1}{s} \sum_{b \in B} \min _{a \in A}\|b-a\|
\end{gathered}
$$

where $\mathrm{R}$ and $\mathrm{S}$ are the number of $\mathrm{A}$ and $\mathrm{B}$ elements respectively.

\subsubsection{Recognition algorithm of human motion based on hidden Markov decision}

Action identification includes the valuation process and learning process, the use of multiple sets of training data, while training Markov model parameters, then get $\lambda_{i}=\left(\pi_{i}, A_{i}, B_{i}\right), \mathrm{i}=1, \ldots ., \mathrm{n}$, Calculate the probability of the sequence to be identified, $\mathrm{P}\left(o \mid \lambda_{\mathrm{i}}\right), \mathrm{i}=1, \ldots, \mathrm{n}$, And the determination result of the selection probability is the action recognition result. The initial model parameter is set to:

The criteria are:

$$
\pi_{\mathrm{i}} \approx 1 / N, \sum_{i} \pi_{i}=1, a_{i j} \approx 1 / N, \sum_{j} a_{i j}=1, b_{j k} \approx 1 / M, \sum_{k} b_{j k}=1 \text { result }=\arg \operatorname{maxP}\left(o \mid \lambda_{\mathrm{i}}\right)
$$

\subsection{Improved DTW-RANSAC Evaluation Model}

\subsubsection{Dynamic Time Warping DTW algorithm}

The Dynamic Time Warping[14]algorithm is commonly used in the field of speech recognition. This article will be used in rehabilitation training process. DTW is a regular algorithm, using a non-linear technology. Through this technology can be structured and measured distance calculation combined. $M$ is the template time axis, $\mathrm{n}$ is the test time axis, in order to calculate the $\mathrm{m}$ and $\mathrm{n}$ non-linear mapping using a regular function, the formula is as follows: 


$$
\mathrm{D}=\min _{\phi\left(\mathrm{i}_{\mathrm{m}}\right)} \sum_{i_{m}=1}^{N} d\left(T\left(i_{n}\right), R\left(\phi\left(i_{n}\right)\right)\right)
$$

The distances between $\mathrm{m}$ and $\mathrm{n}$ are given by parameter $\mathrm{D}$ at the optimal time. The DTW algorithm is used to find the optimal matching path of two vector functions with the smallest vector distance. The algorithm is utilized to calculate the distance between the two vector data. The similarity between the two test vectors is also guaranteed. Match the distance between the two arrays using this function, respectively, with A, B said two arrays, I, J is the length of two arrays, $r$ is the size of the matching window. The size of $r$ takes $1 / 10$ to $1 / 30$ of the array. The return value of the function is the matching distance between $m$ and $n$, and the minimum distance is obtained by dynamic programming. If the return value is -1.0 , the array length is too large. DTW algorithm flow chart is shown in Fig. 2.

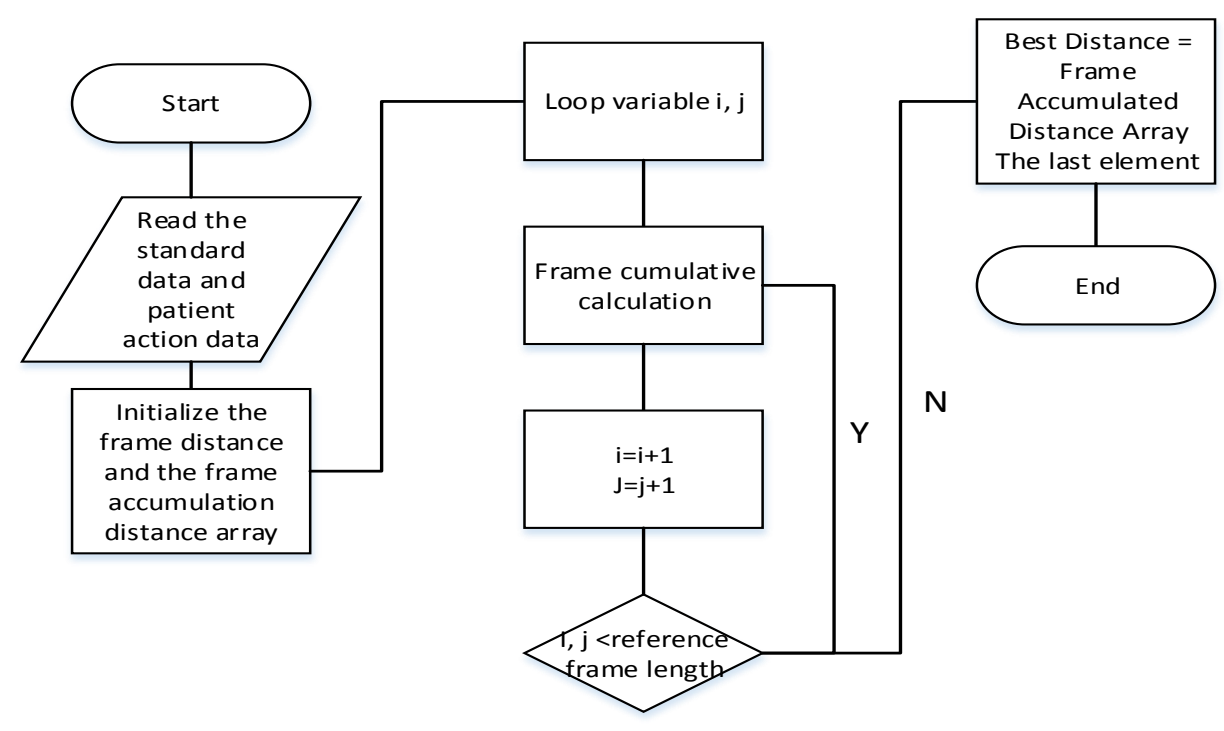

Fig. 2. Uses the DTW algorithm flow chart.

\subsubsection{Evaluation of similarity of motion flow in DTW-RANSAC algorithm}

In the process of rehabilitation, the patient action matching DTW-RANSAC evaluation model; the rehabilitation trainer's action information is set to $X$, the patient's rehabilitation action information is set to $\mathrm{Y}$, the patient and standard action sent to the DTW and RANSAC algorithm The results of the similarity evaluation of motion flow are $x, y$. Moreover, the DTW-RANSAC algorithm also sets two parameters, namely DTW algorithm to assess the credibility of a and RANSAC algorithm to assess the credibility of $b$, the specific algorithm flow shown in Fig. 3.

Rehabilitation training action flow similarity can be based on DTW algorithm, RANSAC algorithm to evaluate the results to write the specific formula:

$$
\text { Similarity D }=(a \times x+b \times y) \times 100 \%
$$

where $\mathrm{D}$ is the final similarity of the action flow $Y$ to $X$;

The similarity between $X$ for the motion stream $Y$ realized by the DTW algorithm is denoted by $x$;

The similarity of $X$ for the motion stream $Y$ realized by the RANSAC algorithm is denoted by $y$;

$\mathrm{a}$ is the reliability of the DTW algorithm, $\mathrm{b}$ is the RANSAC algorithm confidence, and $0 \leq a, n \leq 1, m+n=1$.

In motion flow similarity evaluation mode, the values of $a$ and $b$ are set to $a=0.5, b=0.5$. 
DTW-RANSAC is an algorithm that can obtain valid sample data. When the test data and standard data are sent to DTW and RANSAC, two similarity evaluation results are generated, which enhances the accuracy of similarity evaluation results.

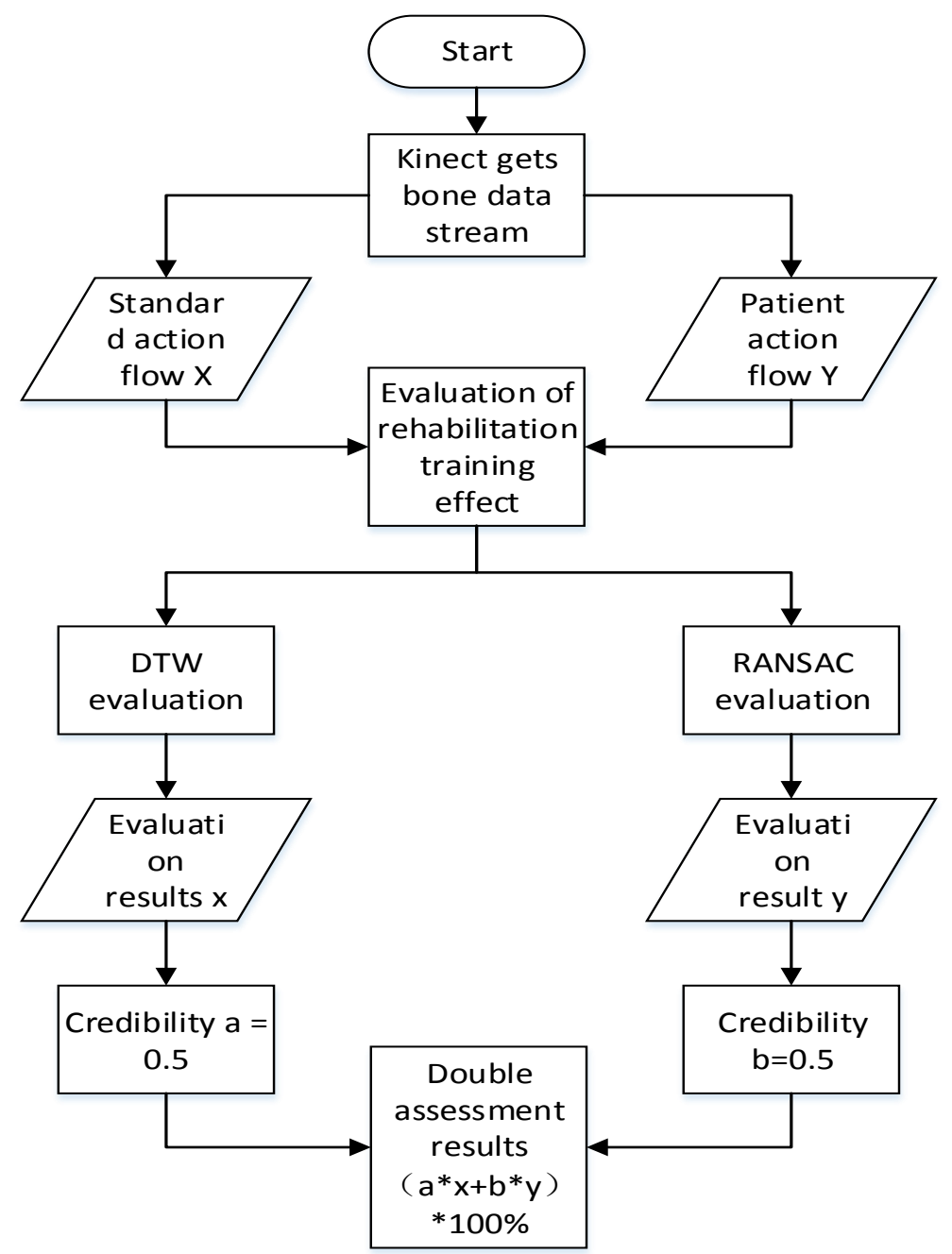

Fig. 3. Dual evaluation based on DTW algorithm and RANSAC algorithm.

\section{System Implementation}

\subsection{Dystem Structure}

Rehabilitation training experts record standard action posture generation rehabilitation training standard action. Patients with rehabilitation training, the system records the patient's action data flow and standard action to match, and the results back to the doctor, the doctor through the rehabilitation training results timely adjustment of rehabilitation training courses, patients can also know their own rehabilitation training and the recovery of their own condition Happening. The system uses a case is shown in Fig. 4.

The user is to use the doctor or the patient side is required to verify the login, if the successful entry into the system main page, if the failure will need to re-login to the patient side, for example, into the main system, the patient selected rehabilitation training, The training results of the patients are matched with the standard template. When the training is completed, the training results are uploaded to the doctor's side. The doctor can view the evaluation of the patient's training results, and then provide the patients with rehabilitation programs and suggestions according to the information, At the same time the two clients also 
have the doctor-patient communication function, doctors and patients can communicate in real time, to explore the disease, greatly facilitate the exchange between doctors and patients to speed up the patient's body recovery rate.

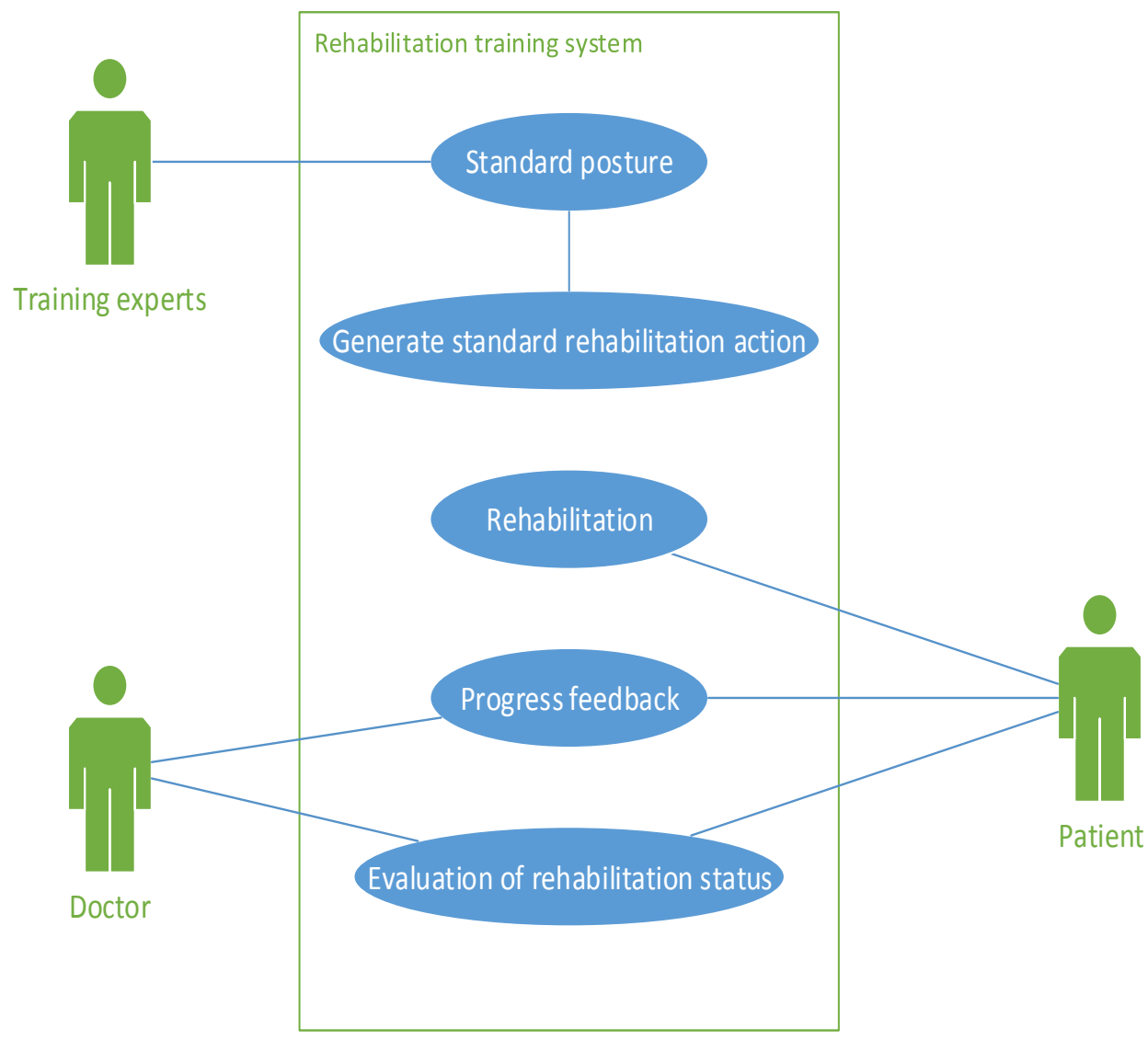

Fig. 4. Use case diagram.

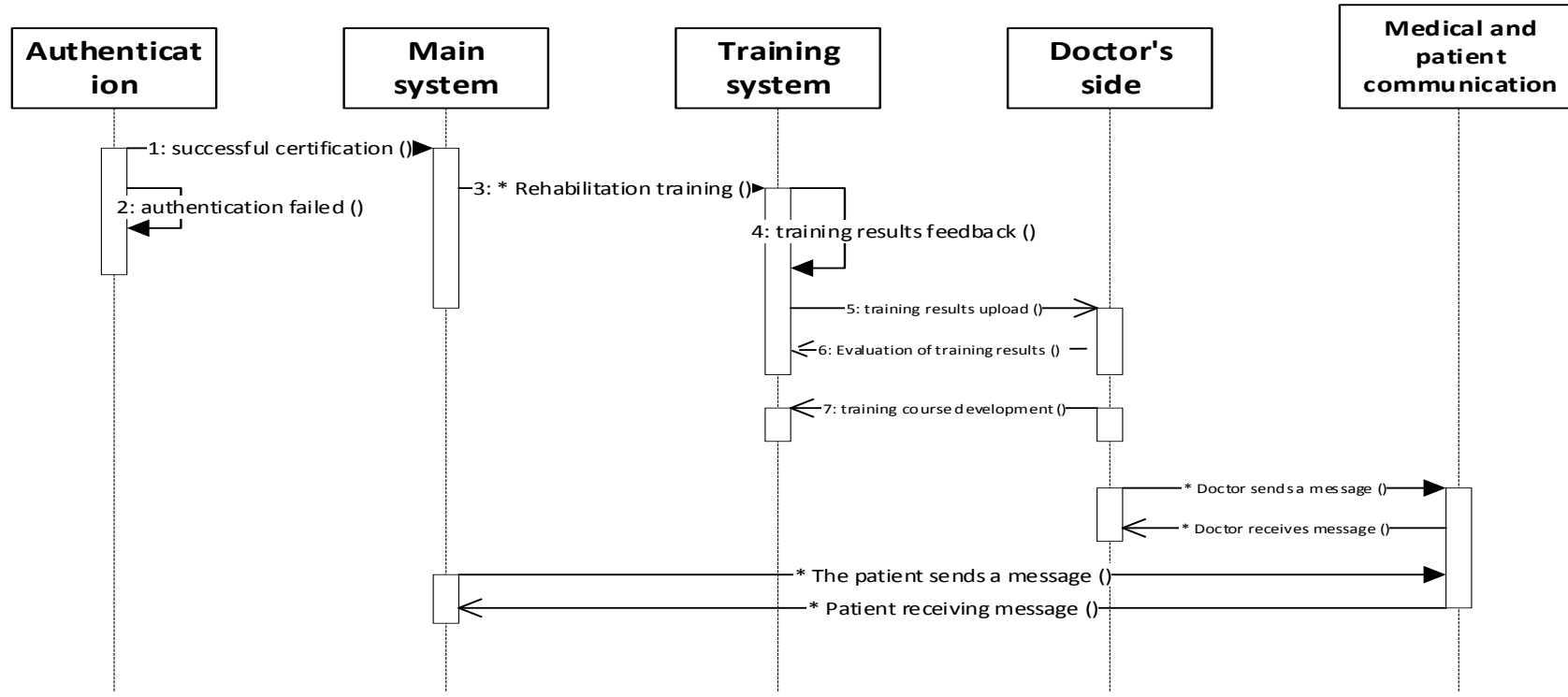

Fig. 5. Timing diagram.

\subsection{Development Process}


The system workflow is designed as follows: first, using the Standard template action of the Kinect recording rehabilitation trainer, the standard action module is used to store the rehabilitative trainer's normal action in the database, and the module generates the standard template by using the data of the Kinect to collect the movement skeleton node of the rehabilitation trainer. In the patients with rehabilitation training, the Kinect collected real-time bone node data into the training action module, and then the training action module of the patient's action and standard action module of the rehabilitation trainer recorded in the standard action of the comparison, give the next step action tips. The doctor uses information feedback and evaluation module to collect the movement data of the patients in the rehabilitative action, and to score the data of the patients's rehabilitation training, and the specific work flow chart is shown in Fig. 6.

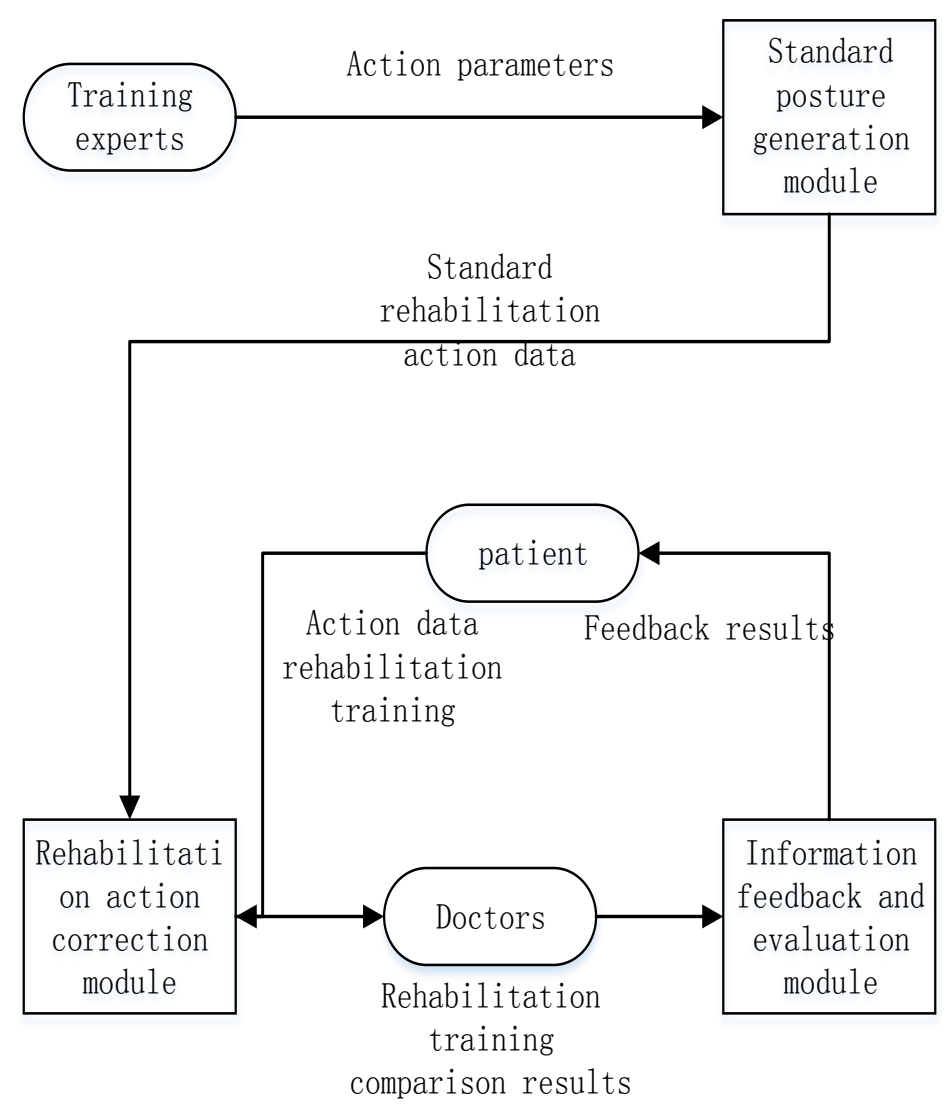

Fig. 6. System implementation flow chart.

\subsection{System Running}

The patient's main interface can be easily trained by various functions such as menu selection and start training. At present, the training of the arm can be realized. The user can start the training by selecting "Start Training" in the "Training" menu. Training items can be fixed by "Training Item Setting", and in the "Training Progress Check" Check your own training schedule. The user will need to be able to view the training of each training program by selecting the menu "Training Course Settings" and download a new training course to study. The user can set and update their personal information via the "Personal Information settings" menu. This information will be displayed to the doctor. The doctor can be more targeted to provide patients with rehabilitation programs and recommendations, is conducive to the recovery of patients. 


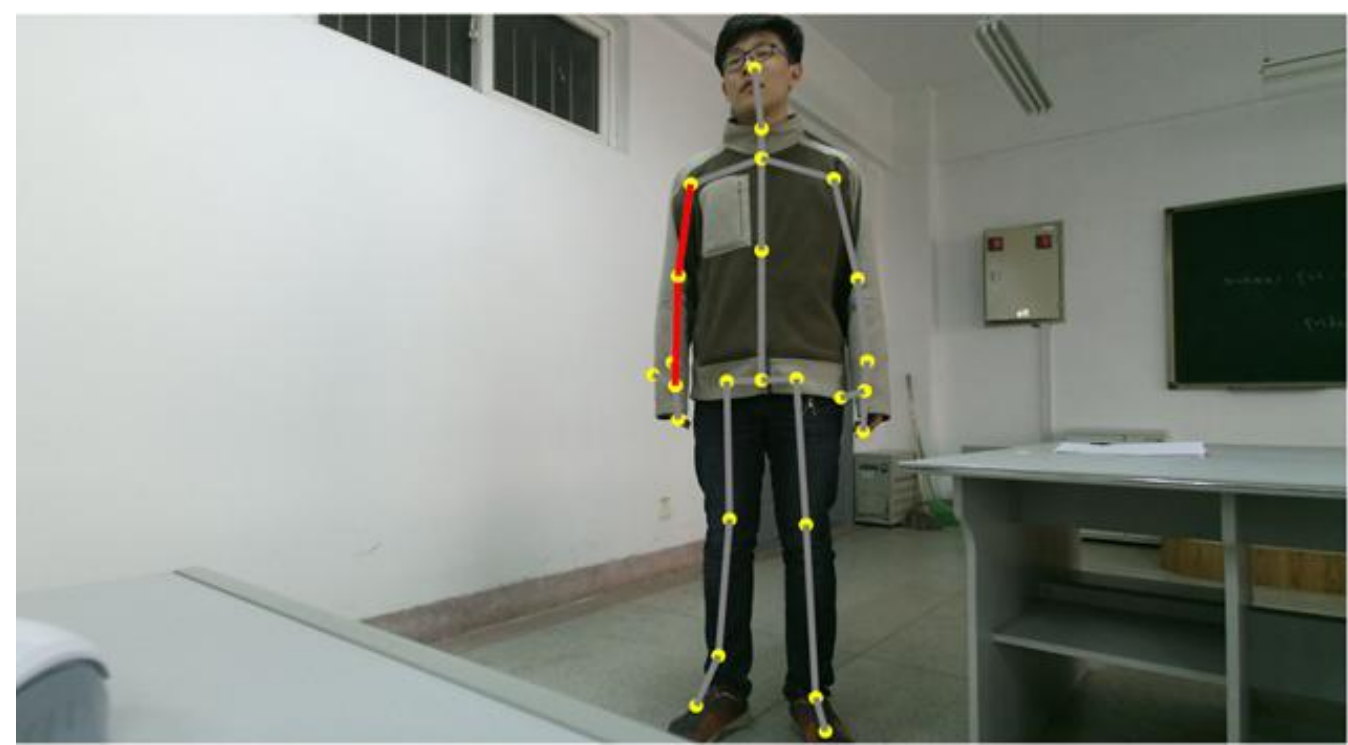

Please lift your arm

Fig. 7. Arm rehabilitation training begins.

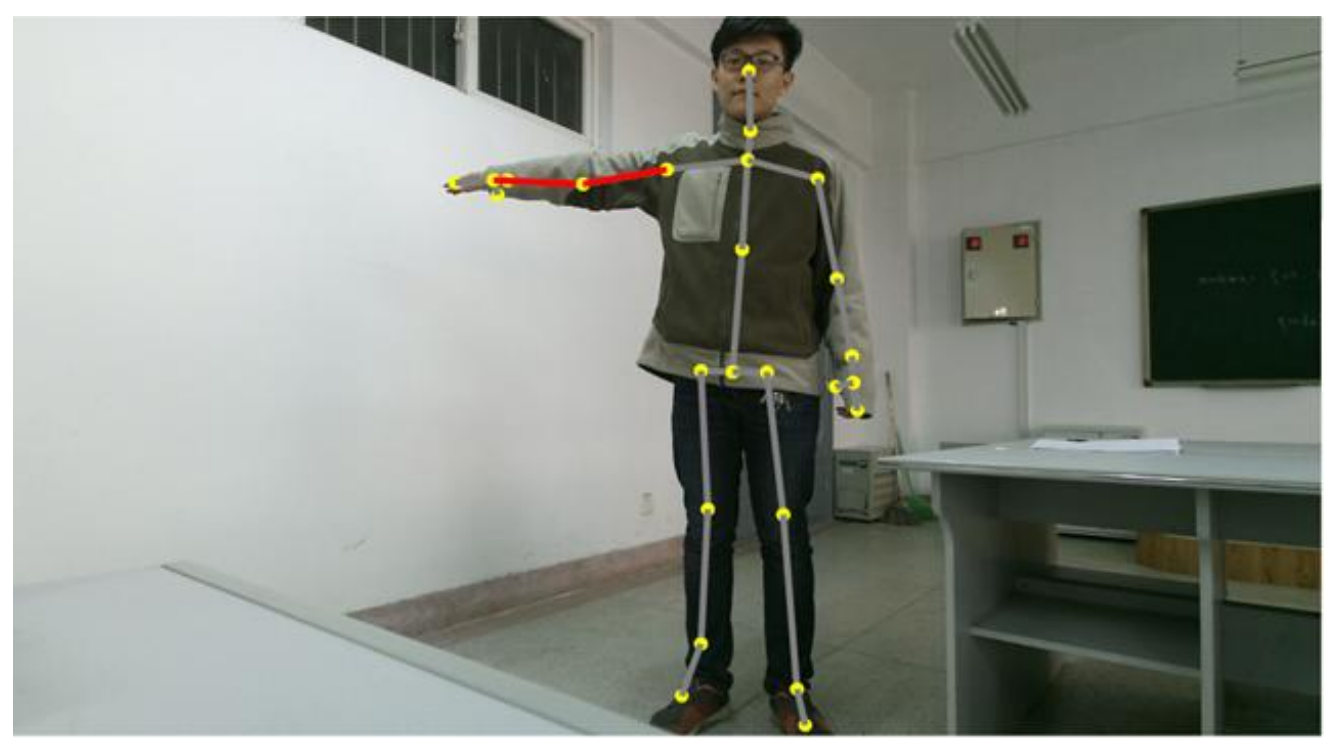

Put down your arm

Fig. 8. End of arm rehabilitation training.

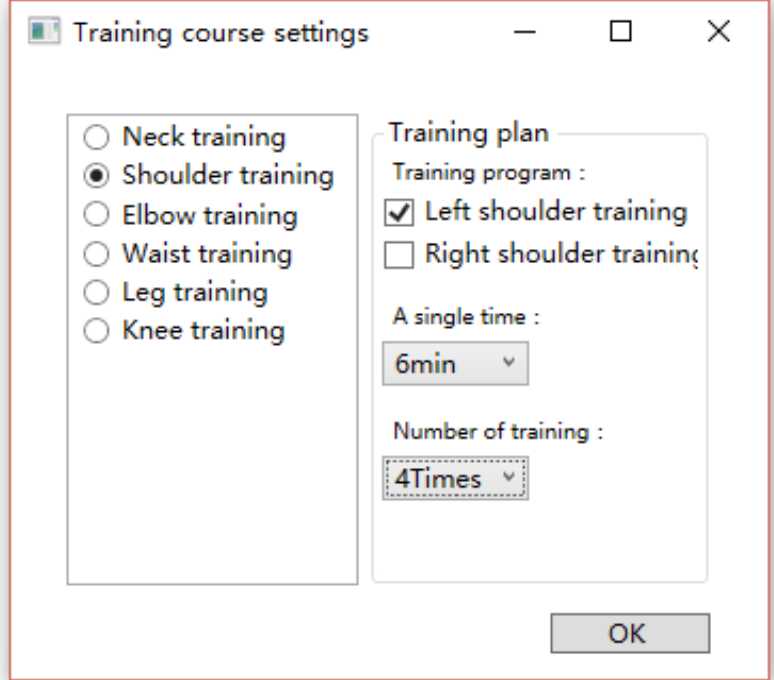

Fig. 9. Rehabilitation training course settings interface. 
Through the training of the project settings, the patient can customize their own training according to their own time and the total number of training, as showed in Fig. 9.

\section{Test}

The hardware environment of this paper is as follows:

CPU: Intel Corei7-7700; RAM: 16GB; hard drive: 1T; GPU: GTX850; Kinect 2.0; and operating under the Windows10 operating system. Compared with the traditional DTW algorithm or the RANSAC algorithm and the improved DTW-RANSAC evaluation model in the system, the relevant test data are shown in Table 1 and Figure 10, and the correct rate of the patients is matched by the DTW algorithm or the RANSAC algorithm. $75 \%$, the correctness of the action matching can be increased to about $85 \%$ after using the DTW-RANSAC evaluation model, which shows that the DTW-RANSAC algorithm improves the matching accuracy of the action.

Table 1. DTW Algorithm ,RANSAC Algorithm and DTW-RANSAC Algorithm Evaluation Results

\begin{tabular}{|c|c|c|c|c|c|c|c|c|c|c|}
\hline & $\begin{array}{l}\text { patient } \\
1\end{array}$ & $\begin{array}{l}\text { Patient } \\
2\end{array}$ & $\begin{array}{l}\text { Patient } \\
3\end{array}$ & $\begin{array}{l}\text { Patient } \\
4\end{array}$ & $\begin{array}{l}\text { Patient } \\
5\end{array}$ & $\begin{array}{l}\text { Patient } \\
6\end{array}$ & $\begin{array}{l}\text { Patient } \\
7\end{array}$ & $\begin{array}{l}\text { Patient } \\
8\end{array}$ & $\begin{array}{l}\text { Patient } \\
9\end{array}$ & $\begin{array}{l}\text { Patient } \\
10\end{array}$ \\
\hline DTW & $80 \%$ & $90 \%$ & $70 \%$ & $60 \%$ & $64 \%$ & $70 \%$ & $80 \%$ & $66 \%$ & $73 \%$ & $92 \%$ \\
\hline RANSAC & $75 \%$ & $86 \%$ & $70 \%$ & $57 \%$ & $60 \%$ & $74 \%$ & $78 \%$ & $70 \%$ & $75 \%$ & $90 \%$ \\
\hline $\begin{array}{c}\text { DTW-RAN } \\
\text { SAC }\end{array}$ & $90 \%$ & $100 \%$ & $85 \%$ & $80 \%$ & $82 \%$ & $80 \%$ & $90 \%$ & $84 \%$ & $87 \%$ & $98 \%$ \\
\hline & $\begin{array}{l}\text { patient } \\
11\end{array}$ & $\begin{array}{l}\text { patient } \\
12\end{array}$ & $\begin{array}{l}\text { patient } \\
13\end{array}$ & $\begin{array}{l}\text { patient } \\
14\end{array}$ & $\begin{array}{l}\text { patient } \\
15\end{array}$ & $\begin{array}{l}\text { patient } \\
16\end{array}$ & $\begin{array}{l}\text { patient } \\
17\end{array}$ & $\begin{array}{l}\text { patient } \\
18\end{array}$ & $\begin{array}{l}\text { patient } \\
19\end{array}$ & $\begin{array}{l}\text { Patient } \\
20\end{array}$ \\
\hline DTW & $59 \%$ & $60 \%$ & $88 \%$ & $95 \%$ & $80 \%$ & $71 \%$ & $90 \%$ & $74 \%$ & $65 \%$ & $80 \%$ \\
\hline RANSAC & $62 \%$ & $58 \%$ & $85 \%$ & $97 \%$ & $83 \%$ & $70 \%$ & $92 \%$ & $73 \%$ & $67 \%$ & $76 \%$ \\
\hline $\begin{array}{c}\text { DTW-RAN } \\
\text { SAC }\end{array}$ & $68 \%$ & $80 \%$ & $97 \%$ & $99 \%$ & $90 \%$ & $84 \%$ & $98 \%$ & $85 \%$ & $78 \%$ & $96 \%$ \\
\hline
\end{tabular}

\section{Comparison of action matching results}

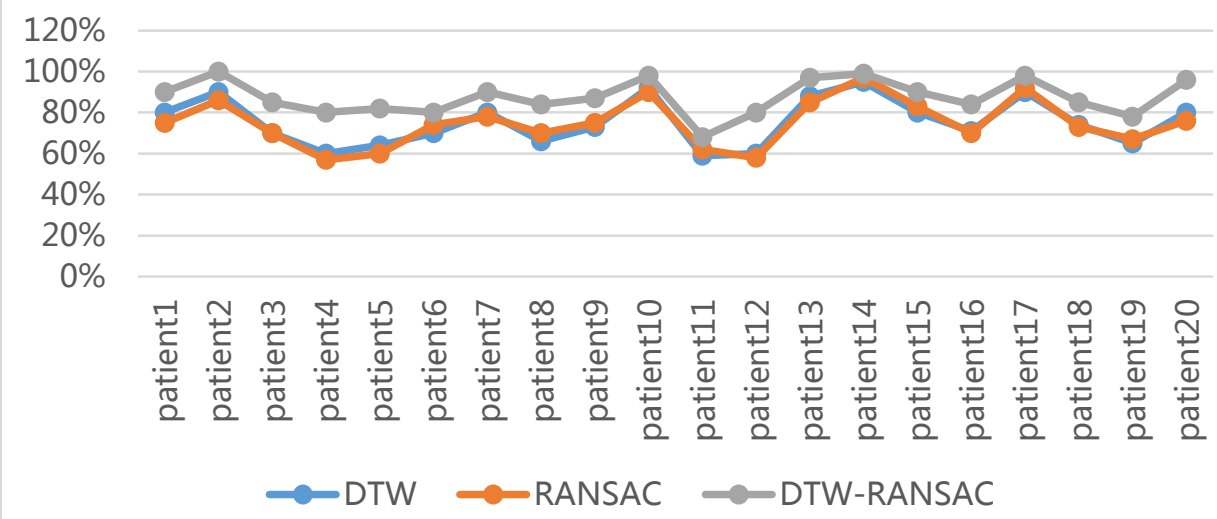

Fig. 10. DTW algorithm ,RANSAC algorithm and DTW-RANSAC algorithm comparison chart.

\section{Conclusion}

In the traditional rehabilitation training process, doctors are required to face rehabilitation training for patients. The principal work of this paper is to provide human motion data through Kinect equipment, 
collect the standard action of rehabilitation trainer, establish standard template database, and provide rehabilitation training for patients. Patients can choose their own rehabilitation training, rehabilitation training results for doctors to view, and timely adjustment of rehabilitation training courses, the patient family can understand the patient's condition in real time. As long as the patient's home computer, Kinect somatosensory equipment and the network can be through the system for rehabilitation training. This paper presents an improved DTW-RANSAC action evaluation model. This model proves its feasibility by experiment, the author will continue to explore the subject, so that more parts of the body can use the system for rehabilitation.

\section{References}

[1] Seonhong, H., Chung-Ying, T., \& Alicia, M. K. (2017). Feasibility study of using a microsoft kinect for virtual coaching of wheelchair transfer techniques. Biomedical Engineering/Biomedizinische Technik.

[2] Auvinet, E., Multon, F., Manning, V., Meunier, J., \& Cobb, J. P. (2016). Validity and sensitivity of the longitudinal asymmetry Index to detect gait asymmetry using microsoft kinect data. Gait \& Posture.

[3] Rafael, C., Alberto, M., Isabel, G., \& Joaquín, G. H. (2017). Kinect as an access device for people with cerebral palsy: A preliminary study. International Journal of Human - Computer Studies.

[4] Namitha, N., Vaitheeswaran, S. M., Jayasree, V. K., \& Bharat, M. K. (2016). Point cloud mapping measurements using kinect RGB-D sensor and kinect fusion for Visual odometry. Procedia Computer Science.

[5] Pierre, P., Hubert, P. H., Shum, A. S. L. P., \& Franck, M. (2016). Validation of an ergonomic assessment method using Kinect data in real workplace conditions. Applied Ergonomics.

[6] Qin, C. L., Song, A. G., Wu, C. C, Liu, Y. Q., \& Jiang, G. H. (2017). Study on interactive scene of rehabilitation robot based on Unity3D and Kinect. Journal of Instrumentation.

[7] Yang, W. l., Wang, J., Xia, B., \& Xie, H. (2017). Research on evaluation system of rehabilitation of lower limb based on Kinect. Journal of Sensors and Microsystems.

[8] Yang, Y. (2012). Based on Kinect-based community home rehabilitation training system. Based on Kinect Sensor Intelligent Wheelchair Gesture Control System Design and Implementation.

[9] Zhou, J., Pan, J. J., Tong, J., Liu, L., \& Pan, Z. (2013). Using Kinect to reconstruct the 3D human body quickly. Journal of Computer Aided Design and Graphiology.

[10] Huttenlocher, D P, Klanderman, G. A., \& Rucklidge, W. J. (1993). Comparing images using the hausdorff distance. Pattern Analysis and Machine Intelligence IEEE Transactions.

[11] Dubuisson, M P., \& Jain, A. K. (1994). A modified hausdorlf distance for object matching. Proceedings of the 12th IAPR International Conference on IEEE 1994.

[12] GAI-Naymat, S. C., \& Taheri, J. (2012). SrseDTW; A novel approach to speed up dynamic time warping. Proceedings of the 2012 Australasian Date Mining.

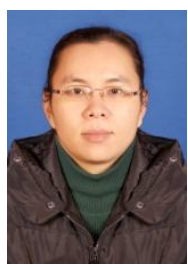

Xiao Nan Shi received her M.E in computer technology from Xi'an University of Science and Technology, Shaanxi, China. Her research interests are computer graphics and virtual reality.She has number of publication in International Journals and conferences.

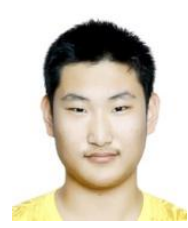

Zi Tong Wang studied at the Department of Software Engineering, Xi'an University of Science and Technology, Shaanxi, China. His research interests are large data and cloud computing, which he conducted in the second to third year undergraduate, won the national innovation and entrepreneurship competition national support. 